\title{
Jupiter's and Saturn's convectively driven banded jets in the laboratory
}

\author{
P. L. Read, Y. H. Yamazaki, S. R. Lewis, P. D. Williams, and K. Miki-Yamazaki \\ Department of Physics, University of Oxford, Oxford, UK
}

J. Sommeria, H. Didelle, and A. Fincham

Laboratoire des Ecoulements Geophysiques et Industriels (LEGI) Coriolis, Grenoble, France

[1] The banded patterns of cloud and wind are among the most striking features of the atmospheres of Jupiter and Saturn, but their dynamical origin remains poorly understood. Most approaches towards understanding zonation so far (also in the terrestrial oceans) have used highly idealized models to show that it might originate from dynamical anisotropy in a shallow turbulent fluid layer due to the planetary $\beta$-effect. Here we report the results of laboratory experiments, conducted on a 14-m diameter turntable, which quantitatively confirm that multiple zonal jets may indeed be generated and maintained by this mechanism in the presence of deep convection and a topographic $\beta$-effect. At the very small values of Ekman number $\left(\leq 2 \times 10^{-5}\right)$ and large local Reynolds numbers ( $\geq 2000$, based on jet scales) achieved, the kinetic energy spectra suggest the presence of both energycascading and enstrophy-cascading inertial ranges in addition to the zonation near twice the Rhines wave number. INDEX TERMS: 0343 Atmospheric Composition and Structure: Planetary atmospheres (5405, 5407, 5409, 5704, 5705, 5707); 3314 Meteorology and Atmospheric Dynamics: Convective processes; 3379 Meteorology and Atmospheric Dynamics: Turbulence; 4528 Oceanography: Physical: Fronts and jets; 5707 Planetology: Fluid Planets: Atmospheres - structure and dynamics.

\section{Introduction}

[2] The cloud bands on Jupiter and Saturn are associated with multiple zonal jets of alternating sign extending to the polar regions [Limaye, 1985; Simon, 1999; Sanchez-Lavega et al., 2000; Porco et al., 2003]. Similar, though less apparent, zonation has also been observed in the terrestrial ocean [Nowlin and Klinck, 1986] and eddy-resolving ocean GCMs [Webb, 2000; Galperin et al., 2004]. Until recently, quantitative understanding of zonation due to the planetary $\beta$-effect has been based principally on high resolution numerical simulations of 2-D or geostrophic turbulence in stirred fluids, but such models are highly idealized and take little account of vertical structure. Laboratory experiments provide alternative means of study, though previous investigations [Condie and Rhines, 1994; Bastin and Read, 1998] have been unable to access regimes at sufficiently high Reynolds number to demonstrate nonlinear zonation convincingly.

[3] Most previous numerical studies have investigated an idealized uniform shallow fluid layer and have concentrated on capturing the nonlinear interactions and cascades characteristic of quasi-2D turbulence, either unforced and decaying with time [e.g., Williams, 1978; Vallis and Maltrud, 1993; Yoden and Yamada, 1993; Huang and Robinson, 1998] or continuously forced either by stochastic eddies [Williams, 1978; Huang et al., 2001] or wind stress and irregular bottom topography [Treguier and Hua, 1988]. Multiple zonal jets have been shown to emerge under various circumstances in such models, typically on a lateral scale (the so-called 'Rhines [1975] scale' $L_{R}$ ) on the order where $U$ is a typical horizontal velocity scale. Where the turbulence is decaying with time in a dissipative flow, the number and scale of the jets is non-stationary, though the external radius of deformation $L_{D} \sim \sqrt{g D} / f$ (where $f$ is the Coriolis parameter and $D$ the fluid depth) may also be of significance in shallow-water flows [Cho and Polvani, 1996]. The latter scale (or its equivalent in a stratified fluid) is also likely to be important in baroclinic flows, more typical of planetary atmospheres [Panetta, 1993]. Indeed Gierasch et al. [1986] noted that $L_{D}$ and $L_{R}$ may be of a similar magnitude at some latitudes on Jupiter. Uncertainties in the respective roles of barotropic and baroclinic effects, and the inability so far of fully 3-D models of the Jovian atmospheres to reproduce their banded zonal dynamics without ad hoc forcing, indicate a strong need to gain access to the appropriate parameter regimes which allow the possibility of fully-developed 3-D effects in a real fluid. In the context of laboratory fluid dynamics, this generally implies a need to carry out experiments on a very large scale, in order to ensure that, when the horizontal scale of the apparatus $L \gg L_{R}$, the Reynolds number defined in terms of $L_{R}\left(\operatorname{Re}_{R} \sim U L_{R} / \nu\right.$, where $\nu$ is the kinematic viscosity of the fluid), is large $(\geq 2000)$ and that the magnitudes of the Ekman numbers $\left(E_{h}=\nu / f L^{2}\right.$ and $\left.E_{v}=\nu / f D^{2}\right)$ are very small.

\section{Experiment}

[4] Our experiments were performed on the $14 \mathrm{~m}$ diameter Coriolis turntable in Grenoble, France, as illustrated in Figure 1. Convective forcing was applied by gently spraying dense water as described in Section 2.1. For the two main experiments to be presented, the rotation period was set to 40 seconds and the buoyancy flux of the forcing, $F_{B}=(\Delta \rho / \rho) g F_{V}$, was $4.6 \times 10^{-8} \mathrm{~m}^{2} \mathrm{~s}^{-3}$, where $F_{V}$ is the volume flux of the spray. The horizontal velocity fields were 


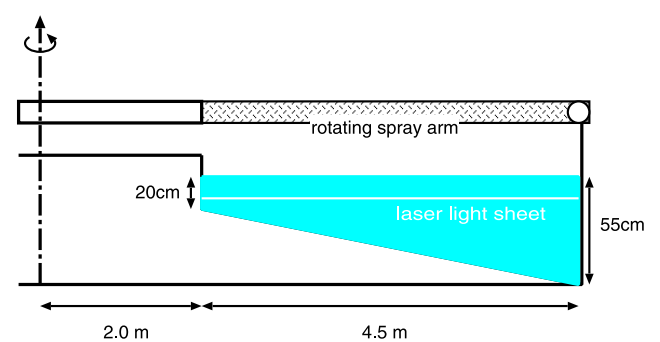

Figure 1. Schematic cross-section of the circular container, showing the rotating spray arm used to provide continuous convective forcing. The entire apparatus is set on a circular turntable.

obtained from plan-view images as described in Section 2.2. A dynamically equivalent $\beta$-effect was obtained by imposing a conical slope at the bottom. The slope was implemented with a stretched sheet, which could be removed for additional experiments with a flat bottom.

\subsection{Convective Forcing}

[5] The cylindrical tank was filled with a homogeneous layer of salty water of density $1.022 \times 10^{3} \mathrm{~kg} \mathrm{~m}^{-3}$, in which neutrally buoyant particles were suspended to enable flow visualization and measurement. Continuous convective forcing across the whole tank was applied by spraying denser (saltier) water from a rotating arm above the tank onto the free surface. The fine shower, which was sprayed at uniform mean intensity per unit area with nozzles carefully arranged to produce a minimal direct disturbance of the free upper surface, led to the continuous generation of small-scale downwelling plumes. These plumes excited small-scale convective cells and vortices, which then interacted to generate larger-scale structures subject to the rotation with topographic $\beta$.

\subsection{Visualization and Data Acquisition}

[6] Both the main and spray water were mixed with neutrally-buoyant beads, and the main fluid was illuminated by a horizontally spreading argon laser sheet through a window in the outer wall. The vertical location of the light sheet was controlled by the main image acquisition computer for supplementary multiple level measurements to verify the vertical uniformity of the flow. Two digital video cameras were mounted $4 \mathrm{~m}$ above the surface to record the images of particle distribution. A higher resolution camera with $1024 \times$ 1024 pixels covered a central $2.5 \mathrm{~m}$ square region of the annular tank, while a wide-angle camera with $768 \times$ 484 resolution covered the entire radial span of the annular channel with a rectangular field of view of $4.5 \mathrm{~m} \times 3.2 \mathrm{~m}$. The images from these cameras were translated into $98 \times 98$ and $94 \times 58$ point velocity fields respectively by CIV analyses [Fincham and Spedding, 1997]. The vertical density profile was also monitored by periodic insertion of a conductivity probe. All data acquisition instruments, including 3 PCs for the 3 devices, and up to 6 operators were located on a co-rotating radial observation bridge over the tank.

\section{Results}

[7] To diagnose the anisotropy of the evolving flow, the velocity fields were decomposed into radial and zonal components in $(r, r \theta)$ coordinates, where $r$ is the radius and $\theta$ the azimuthal angle in radians, as shown in Figure 2a. The well-developed horizontal flow is dominated by eddies of various sizes, but there is also a clear indication of zonation. Figure $2 b$ shows the temporal fluctuation of the mean zonal flow 3 hours after the initial growth of the kinetic energy has saturated. The formation of sustained alternating jets is evident in the sloping bottom experiment, although there is substantial meandering of these jets during this period and occasional splitting and mergers.

[8] Figure 2c shows the initial development for the flat bottom case, in which kinetic energy saturated at $2600 \mathrm{~s}$. The domain-filling single-cell circulation, which is more
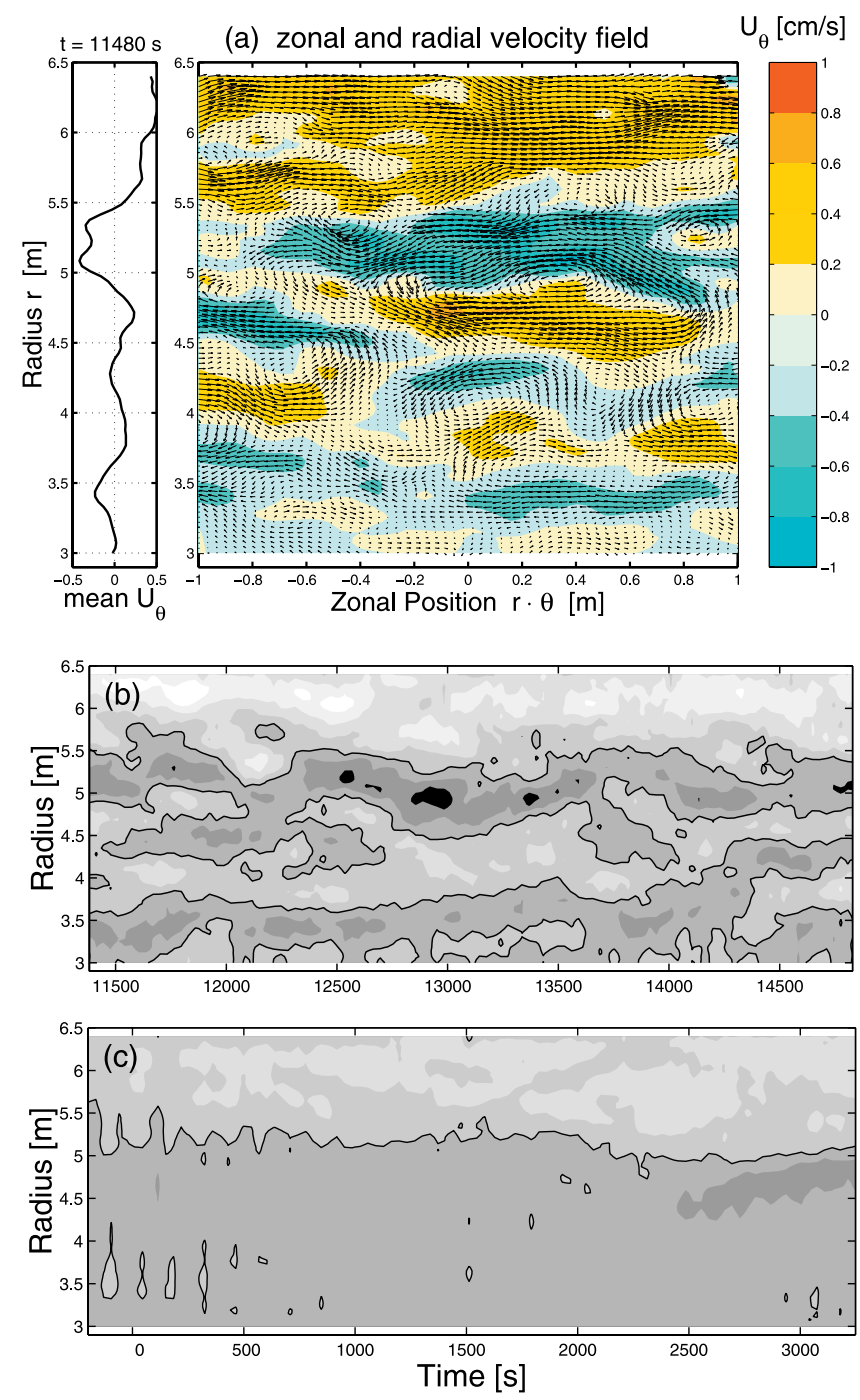

Figure 2. Horizontal velocity field. a, snapshot of the velocity field (right) and spatially averaged zonal velocity (left) from the sloping bottom experiment. b, structure and fluctuation of the mean zonal velocity of the mature flow. The contour interval is $0.2 \mathrm{~cm} \mathrm{~s}^{-1}$ and the solid line denotes the contour of null velocity. The horizontal axis is the elapsed time from when the convective forcing was turned on. c, initial development of the mean flow in the flat bottom experiment (the same notation as b). 
(a)

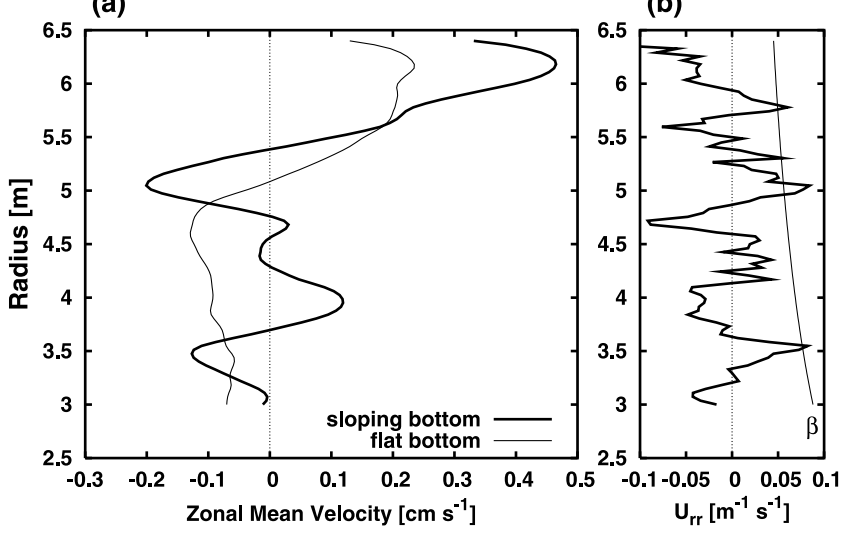

Figure 3. Temporally and spatially averaged zonal flow profile (a) and its second derivative with respect to radius (b) for the sloping bottom experiment. The smooth curve in $\mathrm{b}$ is the profile of topographic $\beta$.

evident in this case, is presumably due at least in part to differential wind stress effects, but we were unable to put a cover over the rotating apparatus to prevent this occurring. Although weaker, this pattern was observed even before the forcing was applied in both experiments as seen, for example, in the leftmost part of Figure 2c.

[9] A canonical zonal flow profile is obtained as the time average of the zonal-mean velocity during the saturated period, and is shown for the sloping bottom experiment in Figure 3, together with its second derivative, $U_{r r}$.

[10] As seen in Figure 3b, the profile of $U_{r r}$ crosses that of topographic $\beta$ by a small margin in several places. Taken at face value, this seems to imply intermittent occurrence of barotropic instability [Kuo, 1949]. In fact, the profile of $U_{r r}$ from instantaneous zonal mean flows often exceeded $\beta$ by a factor of more than 5 . It is of significant interest that similar apparent violations of the stability criterion have been reported for observed Jovian winds. The occurrence of such apparent violations is less surprising, however, in the context of Rossby wave critical layers [Haynes, 1989], a signature of which may be the removal (and even reversal of sign) of a pre-existing lateral potential vorticity gradient in the zonal mean in association with mixing due to breaking and dissipating Rossby waves. We also note that the characteristic jet widths are less than a quarter of the external radius of deformation of 4.5 to $7.4 \mathrm{~m}$.

[11] Figure 4 shows kinetic energy spectra of the zonalmean and eddy components of the flows, calculated from the images obtained by the higher resolution camera. The spectra were first calculated in $x-y$ coordinates as 2-D spectra, and then remapped into total wave number space by integrating over a small interval along the total wave number axis. They were calculated for each snapshot, and averaged over time in Fourier space.

[12] In both cases, the spectral slope is relatively flat below small peaks near $2 k_{\beta}$, with the peak wave number of the eddy energy being slightly higher than that of the zonal mean. The spectrum of medium-scale eddy energy between wave number 15 and $30 \mathrm{~m}^{-1}$ matches well with a $-5 / 3$ slope, which is predicted by the theories of geostrophic and two-dimensional turbulence [Kraichnan, 1967; Sukoriansky et al., 2002]. These results are consistent with the view that small eddies merge and interact to produce larger eddies, and ultimately provide energy to the zonal mean flow. Meanwhile, the slope of the smaller scale energy spectrum is close to -3 , consistent with the classical enstrophy-cascading inertial range of twodimensional and geostrophic turbulence. The "break" in the spectrum at around $k=40 \mathrm{~m}^{-1}$ corresponds to a wavelength of around $15 \mathrm{~cm}$, which is close to the typical scale of individual convective plumes and hence plausibly represents the scale of energy injection. The steep zonalmean flow spectrum is close to the predictions of Huang et al. [2001] and Sukoriansky et al. [2002] for both magnitude and slope.

[13] The spectra of the flat bottom case are harder to interpret. It is interesting, however, that $L_{R}$ associated with the parabolic surface deformation coincides with the width scale of the channel, and the spectral turning point is located at about twice this wave number as in the previous case. The single cell mode in Figure 3 is thus probably
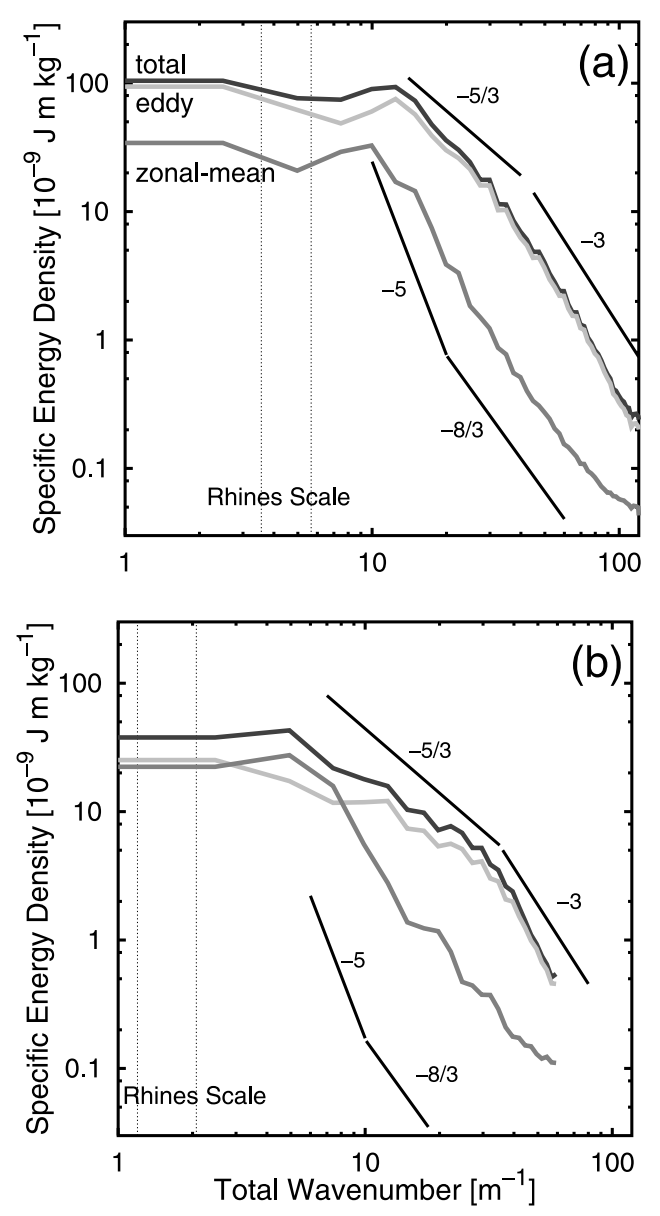

Figure 4. Kinetic energy spectra in total wave number ( $2 \pi$ /wavelength) for (a) the sloping bottom experiment and (b) the flat bottom case. The Rhines wave numbers for the max/min topographic $\beta$ are marked by vertical lines. The solid straight lines are references for the slopes, and the -5 line is the theoretical prediction by Sukoriansky et al. [2002] with $C z=0.5$. 
attributable to the Rhines effect as well as the surface drag.

[14] When the forcing was turned off, the kinetic energy of the sloping bottom experiment started to decay exponentially with a timescale of around $800 \mathrm{~s}$. This timescale agrees well with that due to bottom Ekman friction, $D / \sqrt{\nu f / 2} \approx$ $900 \mathrm{~s}$.

\section{Discussion}

[15] The experiments described herein have enabled us to produce and study a set of zonal jets which are continuously generated near the Rhines scale by the anisotropic modification of an inertially dominated inverse energy cascade in geostrophic turbulence. The influence of the topographic $\beta$-effect is clearly demonstrated in our comparison. The typical Reynolds numbers $\mathrm{Re}_{R}$ obtained with the sloping bottom were much larger (by a factor of at least 10) than typically obtained in previous experiments, enabling the flow to produce fully-developed energy cascades. The horizontal Ekman number, $E_{h}$, was $3 \times 10^{-7}$ and the vertical Ekman numbers, $E_{v}$, for the sloping and flat bottom experiments were $2 \times 10^{-5}$ and $1 \times 10^{-5}$ respectively. The long duration of the experiments (3-6 hours, corresponding to up to around 540 rotation periods) allowed the turbulent flows to develop and to approach an equilibrated state, although it is possible that the jets were still evolving slowly towards the end of the experiment. Numerical experiments [Manfroi and Young, 1999; Huang et al., 2001] suggest that, due to the reduction in efficiency of the inverse energy cascade at the largest scales, the achievement of a dynamic equilibrium may require $\geq 10^{3}$ 'days'.

[16] The multiple jets produced, although not apparently particularly strong or steady (in contrast to those found on Jupiter and Saturn, except perhaps at high latitudes [Porco et al., 2003]), were still easily sufficient to violate the Rayleigh-Kuo stability criterion, even in the timemean. The reason for these differences in morphology from the jets on the outer planets is not immediately apparent, though we speculate (e.g., following Okuno and Masuda [2003]) that this might have something to do with the subsidiary roles of processes on the scales of the radii of deformation. The external deformation radius is much larger than $L_{R}$ in the sloping bottom experiment. In addition, from measurements of the weak density stratification which evolved during the experiment, we estimate the first internal deformation radius $L_{I}=N D / f$ (where $N=\sqrt{-g / \rho(\partial \rho / \partial z)}$ is the buoyancy frequency) to be approximately $10 \mathrm{~cm}$, i.e., comparable to the scale of the small-scale convective plumes and vortices. This large separation of scales is much greater than has been feasible in earlier experiments and, together with the large Reynolds number obtained in our experiments, has evidently allowed the emergence of fully-developed inertial ranges. In addition to their planetary interest, therefore, these experiments also serve a much wider purpose of validating the generic properties (morphology, spectra etc.) of fully-developed geostrophic turbulence as predicted by a range of currently accepted (though often heuristic) theoretical predictions and idealized numerical simulations.
[17] Of course, in the context of the outer planets, many questions remain concerning the possible importance of the detailed vertical structure of Jupiter or Saturn's atmosphere, the uncertain roles of moist convection or other thermodynamic effects in determining the detailed appearance of these planetary jets, not to mention whether convective processes in the deep interiors or in a shallow, near-surface layer are primarily responsible for driving the observed cloud-top circulation. But in either case, it is likely that dynamical interactions between convective motions which are similar to those captured in our experiment play an important role in determining the large-scale organization of the turbulent flow.

[18] Acknowledgments. This project was supported by the HYDRALAB program funded by EC contract Access to Major Research Infrastructures. Additional support was provided by the UK Particle Physics and Astronomy Research Council.

\section{References}

Bastin, M. E., and P. L. Read (1998), Experiments on the structure of baroclinic waves and zonal jets in an internally heated rotating cylinder of fluid, Phys. Fluids, 10, 374-389.

Cho, J. Y.-K., and L. M. Polvani (1996), The emergence of jets and vortices in freely-evolving, shallow-water turbulence on a sphere, Phyis. Fluids, $8,1531-1552$.

Condie, S. A., and P. B. Rhines (1994), A. convective model for the zonal jets in the atmospheres of Jupiter and Saturn, Nature, 367, 711713.

Fincham, A. M., and G. R. Spedding (1997), Low cost, high resolution DPIV for measurement of turbulent fluid flow, Exp. Fluids, 23, 449462.

Galperin, B., H. Nakano, H.-P. Huang, and S. Sukoriansky (2004), The ubiquitous zonal jets in the atmospheres of giant planets and Earth's oceans, Geophys. Res. Lett., 31, L13303, doi:10.1029/ 2004GL019691.

Gierasch, P. J., B. J. Conrath, and J. A. Magalhaes (1986), Zonal mean properties of Jupiter's upper troposphere from Voyager infrared observations, Icarus, 67, 456-483.

Haynes, P. H. (1989), The effect of barotropic instability on the nonlinear evolution of a Rossby-wave critical layer, J. Fluid Mech., 207, 231266.

Huang, H.-P., and W. A. Robinson (1998), Two dimensional turbulence and persistent zonal jets in a global atmospheric model, J. Atmos. Sci., 55, $611-632$

Huang, H.-P., B. Galperin, and S. Sukoriansky (2001), Anisotropic spectra in two-dimensional turbulence on the surface of a rotating sphere, Phys. Fluids, 13, 225-240.

Kraichnan, R. H. (1967), Inertial ranges in two-dimensional turbulence, Phys. Fluids, 10, 1417-1423.

Kuo, H. L. (1949), Dynamic instability of tow-dimensional non-divergent flow in a barotropic atmosphere, J. Meterol., 6, 105-122.

Limaye, S. S. (1985), Jupiter: New estimates of the mean zonal flow at the cloud level, Icarus, 65, 335-352.

Manfroi, A. J., and W. R. Young (1999), Slow evolution of zonal jets on the beta plane, J. Atmos. Sci., 56, 784-800.

Nowlin, W. D., and J. M. Klinck (1986), The physics of the Antarctic circumpolar current, Rev. Geophys., 24, 469-491.

Okuno, A., and A. Masuda (2003), Effect of horizontal divergence on the geostrophic turbulence on a beta-plane: Suppression of the Rhines effect, Phys. Fluids, 15, 56-65.

Panetta, R. L. (1993), Zonal jets in wide baroclinically unstable regions: Persistence and scale selection, J. Atmos. Sci., 50, 2073-2105.

Porco, C. C., et al. (2003), Cassini imaging of Jupiter's atmosphere, satellites and rings, Science, 299, 1541-1547.

Rhines, P. B. (1975), Waves and turbulence on a beta-plane, J. Fluid. Mech., 69, 417-443.

Sanchez-Lavega, A., J. F. Rojas, and P. V. Sada (2000), Saturn's zonal winds at cloud level, Icarus, 147, 405-420.

Simon, A. A. (1999), The structure and temporal stability of Jupiter's zonal winds: A study of the north tropical region, Icarus, 141, 29-39.

Sukoriansky, S., B. Galperin, and N. Dikovskaya (2002), Universal spectrum of two-dimensional turbulence on a rotating sphere and some basic features of atmospheric circulations on giant planets, Phys. Rev. Lett., 89, 124501. 
Treguier, A. M., and B. L. Hua (1988), Influence of bottom topography on stratified quasi-geostrophic turbulence in the ocean, Geophys. Astrophys. Fluid Dyn., 43, 265-305.

Vallis, G. K., and M. E. Maltrud (1993), Generation of mean flows and jets on a beta plane and over topography, J. Phys. Oceanogr., 23, 13461362.

Webb, D. J. (2000), Evidence for shallow zonal jets in the South Equatorial Current region of the southwest Pacific, J. Phys. Oceanogr., 30, 706720 .

Williams, G. P. (1978), Planetary circulations: 1. Barotropic representation of Jovian and Terrestrial turbulence, J. Atmos. Sci., 35, 13991426.
Yoden, S., and M. Yamada (1993), A numerical experiment on twodimensional, decaying turbulence on a rotating sphere, J. Atmos. Sci., $50,631-643$.

S. R. Lewis, K. Miki-Yamazaki, P. L. Read, P. D. Williams, and Y. H. Yamazaki, Atmospheric, Oceanic and Planetary Physics, University of Oxford, Parks Road, Oxford OX1 3PU, UK. (hiro@atm.ox.ac.uk)

H. Didelle, A. Fincham, and J. Sommeria, LEGI Coriolis, 21 rue des Martyrs, F-38000 Grenoble, France. 